\title{
ON A NONLINEAR HYDROMAGNETIC CONVECTION UNDER A ROTATIONAL CONSTRAINT
}

\author{
N. RIAHI
}

(Received 12 September 1979)

\begin{abstract}
Nonlinear hydromagnetic convection in a horizontal layer of fluid rotating about the vertical axis is investigated using the mean field approximation. The boundary layer method is used assuming large Rayleigh number $R$ for different ranges of the Chandrasekhar number $Q$ and the Taylor number $\mathrm{Ta}$. The heat flux $F$ is determined for the value of the horizontal wave number $\alpha$ which maximizes $F$. It is shown that, for certain regions of the parameter space $(R, Q, T a), F$ and $\alpha$ change discontinuously for $T a$ greater than some critical value (given $R$ and $Q$ ). Thus, for $T a$ about this critical value, wave numbers and heat fluxes of two different values will be predicted simultaneously. Also, for certian regions of the parameter space, the field can facilitate convection, but rotation can facilitate convection only for sufficiently large $T a$.
\end{abstract}

\section{Introduction}

The effect on nonlinear Rayleigh-Benard convection of either rotation alone (Hunter and Riahi [7], Riahi [8] and Chan [3], henceforth referred to as I) or a magnetic field alone (Van der Borght et al. [10] and Riahi [9], henceforth referred to as II) has recently been studied, and these studies provide further insights into the subject of nonlinear convection with the effect of rotation and magnetic field acting separately. These studies have confirmed that an impressed magnetic field or rotation can significantly modify the convective flow and the total heat transport. However, contrary to expectations, the linear theory (Chandrasekhar [4]) predicts that when both a magnetic field and rotation act together, they may have conflicting tendencies.

The present study concerns the combined effect of a magnetic field and rotation on nonlinear thermal convection at large Rayleigh numbers and it is hoped that it provides a deeper insight into the subject of hydromagnetic convection in a rotating fluid. 
We shall study the nonlinear problem subject to the so-called mean field equations for the magnetic field, momentum and heat. Briefly, these equations are derived by ignoring the interaction between the fluctuation quantities, but the interaction between the mean and the fluctuation quantities is retained. For a more detailed discussion of these equations and their derivation, we refer to the papers by Herring [5] and Busse [1]. Previous studies of these equations for the case of thermal convection have shown that, for moderate or large values of the Prandtl number $\mathrm{Pr}$, the derived results, as far as the statistical properties of motion are concerned, do not differ appreciably from the experimental results based on the full convection equations.

As in II, we are interested in finding the solution which maximizes the heat transport $F$. It is known from recent studies of Benard convection with and without rotation (Chan [2] and Hunter and Riahi [7]) that the flow that maximizes $F$ subject to the mean field equations is the same as that which gives an upper bound to $F$ in the limit of large $P r$ for the full convection equations. The flow that maximizes $F$ for the mean field equations of hydromagnetic convection is believed to represent adequately the flow which gives an upper bound to $F$ for the full hydromagnetic convection equations at least in the limit of large $\operatorname{Pr}$ and diffusivity ratio $\tau$ (ratio of magnetic diffusivity to thermal diffusivity). The success of the previous upper bound studies of thermal convection, which have compared reasonably well with observation, encouraged us to undertake the present study, which we hope will provide us with a deeper insight into the nature of the combined effects of rotation and magnetic field on nonlinear convection, something which is of prime importance in many geophysical and astrophysical problems.

\section{Governing equations}

We consider a horizontally infinite layer of fluid of depth $d$, bounded above and below. The upper and lower surfaces are maintained at temperatures $T_{0}$ and $T_{0}+\Delta T_{0}$, with $\Delta T>0$, respectively. The fluid is rotating about the vertical with angular velocity $\Omega$ and is permeated by an imposed magnetic field in the vertical direction. The magnetic field, $\mathbf{M}=\left(M_{1}, M_{2}, M_{3}\right)$, can be written as $\mathbf{M}=\overline{\mathbf{M}}+\mathbf{h}$, where a bar above denotes a horizontal average. Since $\overline{\mathbf{M}}$ is only a function of the vertical variable and $\nabla \cdot \overline{\mathbf{M}}=0, \overline{\mathbf{M}}_{3}$ must be a constant and takes on the value of the impressed field. If this value is taken as a unit of the field strength, then $\mathbf{M}=\mathbf{k}+\mathbf{h}$, where $\mathbf{k}$ is the unit vector in the vertical direction. The mean field equations of the hydromagnetic convection are derived from the Boussinesq equations for momentum, magnetic field and heat when all nonlinear terms are neglected with the exception of the one which enters the equation for the horizontally averaged temperature (Busse [1]). The nondimensional steady-state forms of these equations, after eliminating the pressure and horizontal velocity components, are 


$$
\begin{aligned}
\nabla^{2} \hat{S}+\frac{2}{E} \frac{\partial W}{\partial Z}+\tau Q \frac{\partial \hat{C}}{\partial Z} & =0, \\
\frac{\partial \hat{S}}{\partial Z}+\tau \nabla^{2} \hat{C} & =0, \\
\nabla^{4} W+R \nabla_{1}^{2} T+\tau Q \nabla^{2} \frac{\partial h_{3}}{\partial Z}-\frac{2}{E} \frac{\partial \hat{S}}{\partial Z} & =0, \\
\frac{\partial W}{\partial Z}+\tau \nabla^{2} h_{3} & =0, \\
\nabla^{2} T+(1-\overline{W T}+\langle W T\rangle) W & =0,
\end{aligned}
$$

where $W, h_{3}, \hat{S}$ and $\hat{C}$ are the vertical components of velocity, magnetic fluctuation, vorticity and curl of magnetic fluctuation, respectively. Also, $T$ is the deviation of temperature from its horizontal average, $E=v / d^{2} \Omega$ is the Ekman number, $R=\beta g \Delta T d^{3} / \kappa v$ is the Rayleigh number, $Q=\bar{M}_{3}^{2} d^{2} / \mu \rho_{0} v \eta$ is the Chandrasekhar number, $\tau=\eta / \kappa$ is the ratio of magnetic diffusivity to thermal diffusivity, $\mu$ is the magnetic permeability, $\rho_{0}$ is the reference density (constant), $v$ is the kinematic viscosity, $\beta$ is the coefficient of thermal expansion, $g$ is the acceleration due to gravity, angle brackets denote a total volume average over the whole layer, and $\nabla_{1}^{2}$ is the horizontal Laplacian.

We shall rescale our dependent variables so that

$$
\left.\begin{array}{c}
\omega=(F R)^{-\frac{1}{2}} W, \quad \theta=(R / F)^{\frac{1}{2}} T, \\
H=(F R)^{-\frac{1}{2}} \tau h_{3}, \quad S=(F R)^{-\frac{1}{2}} \hat{S}, \quad C=(F R)^{-\frac{1}{2}} \tau \hat{C},
\end{array}\right\}
$$

where $F=\langle W T\rangle$ is the heat flux. The governing equations are now

$$
\begin{aligned}
\nabla^{2} S+(T a)^{\frac{1}{2}} \frac{\partial \omega}{\partial Z}+Q \frac{\partial C}{\partial Z} & =0, \\
\frac{\partial S}{\partial Z}+\nabla^{2} C & =0, \\
\nabla^{4} \omega+\nabla_{1}^{2} \theta+Q \nabla^{2} \frac{\partial H}{\partial Z}-(T a)^{\frac{1}{2}} \frac{\partial S}{\partial Z} & =0, \\
\frac{\partial \omega}{\partial Z}+\nabla^{2} H & =0, \\
\frac{1}{F R} \nabla^{2} \theta+\left(1-\overline{\omega \theta}+\frac{1}{F}\right) \omega & =0,
\end{aligned}
$$

where $T a=4 / E^{2}$ is the Taylor number. 
We shall use the following constraint to determine $F$,

$$
F=\frac{1-R^{-1}\left\langle|\nabla \theta|^{2}\right\rangle}{\left\langle(1-\omega \theta)^{2}\right\rangle}
$$

which is derived by multiplying equation (3e) by $\theta$ and taking the total average over the whole layer.

As in II, the boundary conditions to be considered for free surfaces at $z=0,1$ are

$$
\omega=\frac{\partial^{2} \omega}{\partial Z}=\theta=H=\frac{\partial S}{\partial Z}=\frac{\partial C}{\partial Z}=0 .
$$

The basic equations (3) are separable and admit solution of the form

$$
(\omega, \theta, H, S, C)=\left[\omega_{1}(Z), \theta_{1}(Z), H_{1}(Z), S_{1}(Z), C_{1}(Z)\right] \phi(x, y),
$$

where

$$
\nabla_{1}^{2} \phi=-\alpha^{2} \phi
$$

In equations (6), $\alpha$ is the horizontal wave number and $\phi$ is the planform of the cellular pattern with horizontal scale of the order of $\alpha^{-1}$. The unit subscripts will now be omitted for simplicity, so that

$$
\begin{aligned}
\left(\frac{d^{2}}{d Z^{2}}-\alpha^{2}\right) S+(T a)^{\frac{1}{2}} \frac{d \omega}{d Z}+Q \frac{d C}{d Z} & =0, \\
\frac{d S}{d Z}+\left(\frac{d^{2}}{d Z^{2}}-\alpha^{2}\right) C & =0 \\
\left(\frac{d^{2}}{d Z^{2}}-\alpha^{2}\right)^{2} \omega-\alpha^{2} \theta+Q\left(\frac{d^{2}}{d Z^{2}}-\alpha^{2}\right) \frac{d H}{d Z}-(T a)^{\frac{1}{2}} \frac{d S}{d Z} & =0 \\
\frac{d \omega}{d Z}+\left(\frac{d^{2}}{d Z^{2}}-\alpha^{2}\right) H & =0, \\
\frac{1}{F R}\left(\frac{d^{2}}{d Z^{2}}-\alpha^{2}\right) \theta+\left(1-\omega \theta+\frac{1}{F}\right) \omega & =0
\end{aligned}
$$

with boundary conditions

$$
\omega=\frac{d^{2} \omega}{d Z^{2}}=\theta=H=\frac{d S}{d Z}=\frac{d C}{d Z}=0 \quad \text { at } Z=0,1 .
$$

Equations (7) and (8) must then be solved subject to (4). We shall obtain the solutions by using the boundary layer method, treating $R$ as a large parameter. 


\section{Boundary layer solutions}

\subsection{Rotational and magnetic effects are unimportant}

The range in which rotational and magnetic effects are unimportant is found to be $T a \ll 1$ and $Q \ll 1$. The solution in this case is essentially that given by Howard [6] for $Q=0$ and $T a=0$. The boundary layer structure is unaffected by the field and consists of a nonuniform interior and a thin thermal layer of thickness proportional to $R^{-1 / 3}$ closed to each boundary. The heat flux $F$ is proportional to $R^{1 / 3}$ and $\alpha$ is of order one.

\subsection{Rotational effects are unimportant}

Rotational effects do not become significant immediately after $T a$ becomes nonzero, but they can initially be regarded as small perturbations to the solution without rotation. The range in which rotational effects are unimportant is found to be $T a \ll 1$, and the solution is essentially that given in II for $T a=0$. We shall briefly review the main results derived in II and refer the reader to that paper for details on the subject.

According to the results derived in II, it is appropriate to divide the parameter space into four different regions :

(i) The range $1 \ll Q \ll R^{\frac{1}{2}}$. The solution is qualitatively the same as in the case described in Section 3.1, except that $\alpha$ is now proportional to $Q^{\frac{1}{2}}$. Since $\alpha$ is large, there exists also an intermediate layer of thickness $\alpha^{-1}$. The interior is now uniform. The heat flux $F$ is proportional to $R^{1 / 3}$, but the proportionality factor depends on $Q$ and decreases as $Q$ increases.

(ii) The range $R^{\frac{1}{2}} \ll Q \ll R^{3 / 4}(\log R)$. The boundary layer structure consists of a uniform interior, an inner layer of thickness $Q^{\frac{1}{2}} / \alpha^{2}$, an intermediate layer of thickness $\alpha^{-1}$ and a thermal layer of thickness of order $F^{-1}$. The wave number $\alpha$ and the heat flux are proportional to $R^{1 / 4}$ and $R^{\frac{1}{2}} Q^{-1 / 3}\left(\log Q R^{-\frac{1}{2}}\right.$ ), respectively. (Specifically, $\alpha=(R / 9)^{1 / 4}$.)

(iii) The range $R^{3 / 4}(\log R) \ll Q \ll(R \log R)^{4 / 5}$. This case is essentially that of case (ii), except that the thermal layer merges with the intermediate layer. The quantities $\alpha$ and $F$ are both proportional to $R Q^{-1}\left(\log Q^{3 / 2} R^{-1}\right)$.

(iv) The range $(R \log R)^{4 / 5} \ll Q \ll R$. This case is essentially that of case (iii), except that the thermal layer is now thicker than the intermediate layer. The thickness of the thermal layer, $\alpha$ and $F$ are proportional to $F^{-1}, Q^{1 / 4}$ and $R Q^{-1}\left(\log R Q^{-1}\right)$, respectively.

\subsection{Magnetic effects are unimportant}

Magnetic effects do not become significant immediately after $Q$ becomes nonzero, but they can initially be regarded as small perturbations to the solution without the 
field. The range in which magnetic effects are unimportant is found to be $Q \ll 1$, and the solution is essentially that given in I for $Q=0$. Here we briefly review the main results derived in I and refer the reader to that paper for details on the subject.

It is found to be appropriate to divide the parameter space into three different regions :

(i) The range $1 \ll T a \ll R$. The solution is qualitatively the same as in the case described in Section 3.1, except that $\alpha$ is now proportional to $T a^{1 / 4}$. (Chan in I incorrectly states that $\alpha$ is proportional to $R^{1 / 4}$.) Since $\alpha$ is large, there exists also an intermediate layer of thickness $\alpha^{-1}$. The interior is now uniform. The heat flux $F$ is proportional to $R^{1 / 3}$, but the proportionality factor depends on $T a$ and decreases as Ta increases.

(ii) The range $R \ll T a \ll R^{11 / 10}\left(\log T a R^{-1}\right)$. The boundary layer structure consists of a uniform interior, an inner layer of thickness $T a^{\frac{1}{2}} / \alpha^{3}$, and a thermal layer of thickness of order $F^{-1}$. In addition, there is an intermediate layer of thickness $\alpha^{-1}$ (between the inner layer and the thermal layer) which adjusts the solution to satisfy the correct boundary condition on $H$; such a layer does not exist in the case $Q=0$. We have that

$$
F=2^{4 / 3}(1.062)^{-4 / 3} 5^{-5 / 3} R^{2 / 3} T a^{-1 / 3}\left(\log T a R^{-1}\right)^{1 / 3} \text { and } \alpha=(R / 5)^{1 / 4} .
$$

The expression for $F$ is slightly different from the one obtained in I $(Q=0)$ which is

$$
F=2.3^{-1 / 3}(1.062)^{-4 / 3} 5^{-5 / 3} R^{2 / 3} T a^{-1 / 3}\left(\log T a^{2} R^{-1}\right)^{1 / 3} .
$$

This difference is caused mainly by the intermediate layer which exists only in the case $Q \neq 0$.

(iii) The range $R^{5 / 4}(\log R) \ll T a \ll R^{3 / 2}$. This case is essentially that of case (ii), except that the thermal layer is now thicker than the intermediate layer. In this case $F=(4 / \pi)^{2} 5^{-5 / 2} R^{3 / 2} \mathrm{Ta}^{-1}\left(\log R^{3 / 2} \mathrm{Ta}^{-1}\right)$. The expression for $F$ derived in $\mathrm{I}$ has incorrectly an additional factor of 4 .

\subsection{Magnetic field is dominant}

The solutions for this case are essentially those discussed in Section 3.2, except for the following differences obtained in each of the four different ranges for $Q$ :

(i) The range $1 \ll Q \ll R^{\frac{1}{2}}$. The Taylor number $T a$ must be in the range $1 \ll T a \ll Q^{2}$.

(ii) The range $R^{\frac{1}{2}} \ll Q \ll R^{3 / 4}(\log R)$. The Taylor number $T a$ must be in the range $1 \ll T a \ll Q R^{\frac{1}{2}}$.

(iii) The range $R^{3 / 4}(\log R) \ll Q \ll(R \log R)^{4 / 5}$. The Taylor number Ta must be in the range $1 \ll T a \ll R^{2} Q^{-1}\left(\log Q^{3 / 2} R^{-1}\right)^{2}$.

(iv) The range $(R \log R)^{4 / 5} \ll Q \ll R$. The Taylor number Ta must be in the range $1 \ll T a \ll Q^{3 / 2}$. 


\subsection{Magnetic field is marginally dominant}

(i) The range $1 \ll Q \ll R^{\frac{1}{1}}$. The Taylor number $T a$ is proportional to $Q^{2}$. The rest of the results are essentially the same as those discussed for this range in Section 3.2.

(ii) The range $R^{\frac{1}{2}} \ll Q \ll R^{3 / 4}(\log R)$. The boundary layer structure consists of four distinct regions: the interior, an inner layer of thickness $\varepsilon=T a^{\frac{1}{2}} / \alpha^{3}$, an intermediate layer of thickness $\alpha^{-1}$, and a thermal layer. Without loss of generality, we shall restrict ourselves to the discussion of the boundary layer structure near the lower boundary, since the boundary layer structure near the upper boundary is essentially the same as the one near the lower boundary.

In the interior, $z$ is of order one. The wave number $\alpha$ is supposed to be large (which can be justified a posteriori), so that the convection cells are narrow. Equations (7) then yield

$$
\begin{aligned}
-\alpha^{2} S+(T a)^{\frac{1}{2}} \frac{d \omega}{d Z}+Q \frac{d C}{d Z} & =0, \\
\alpha^{2} C & =\frac{d S}{d Z}, \\
\omega-\alpha^{-2} \theta-\alpha^{-2} Q \frac{d H}{d Z}-(T a)^{\frac{1}{2}} \alpha^{-4} \frac{d S}{d Z} & =0, \\
\alpha^{2} H & =\frac{d \omega}{d Z} \\
-\frac{\alpha^{2}}{F R} \theta+(1-\omega \theta) \omega & =0 .
\end{aligned}
$$

It is assumed that

$$
\alpha^{4} \ll F R, \quad Q \ll \alpha^{4} \quad \text { and } \quad T a \ll \alpha^{6} .
$$

Using (7) and (10) in (9), we find that the interior is uniform and isothermal. The solutions are

$$
\omega=\alpha^{-1}, \quad \theta=\alpha \text { and } H=C=S=0 .
$$

Thus in the interior the vorticity does not modify the velocity field and the fluid motion is essentially vertical. The magnetic field in this region is essentially the same as the imposed external field.

In the inner layer of thickness $\varepsilon$, we define $\psi=z / \varepsilon$ as the variable in this layer. It is assumed that

$$
\alpha^{-1} \ll \varepsilon \ll 1
$$

and the conductive term is not yet important. 
Using (12), the governing equations (7) yield

$$
\begin{aligned}
-S+\frac{T a^{\frac{1}{2}}}{\alpha^{2} \varepsilon} \frac{d \omega}{d \psi}+\frac{Q}{\varepsilon \alpha^{2}} \frac{d C}{d \psi} & =0, \\
\alpha^{2} \varepsilon C & =\frac{d S}{d \psi}, \\
\omega-\alpha^{-2} \theta-\frac{Q}{\alpha^{2} \varepsilon} \frac{d H}{d \psi}-\frac{T a^{\frac{1}{2}}}{\alpha^{4} \varepsilon} \frac{d S}{d \psi} & =0, \\
\alpha^{2} \varepsilon H & =\frac{d \omega}{d \psi}, \\
\omega \theta & =1 .
\end{aligned}
$$

It is then found that we must have the following conditions:

$$
Q \gg \alpha^{2}
$$

and

$$
T a=\beta^{2} \alpha^{2} Q
$$

where $\beta$ is a constant of order one. Using (14b) and (12), we find the following necessary condition which must hold when both rotation and the field are dominant

$$
Q^{3 / 2} \ll T a \ll Q^{2}
$$

We find from (13), after applying matching conditions (matching the solutions to the corresponding solutions in the interior), that as $\psi \rightarrow 0$

$$
\left.\begin{array}{rl}
\omega & =\beta \alpha^{-1} \psi\left(2 \log \psi^{-1}\right)^{\frac{1}{2}}, \\
H & =\beta \alpha^{-3} \varepsilon^{-1}\left(2 \log \psi^{-1}\right)^{\frac{1}{2}}, \\
S & =\exp (-\beta \psi)-\beta \psi^{2}\left(2^{-1} \log \psi^{-1}\right)^{\frac{1}{2}}, \\
c & =-\left[\exp (-\beta \psi)+\beta \psi\left(2 \log \psi^{-1}\right)^{\frac{1}{2}}\right] \alpha^{-2} \varepsilon^{-1} .
\end{array}\right\}
$$

In the intermediate layer of thickness $\alpha^{-1}$, we define $\eta=\alpha z$ as the variable in this layer. Since conductive terms are not yet important, (13e) is still valid in this tayer. We then find from (7), after applying matching conditions (matching the solutions to the corresponding solutions in the inner layer), that the governing equations reduce to

$$
\begin{aligned}
\frac{d C}{d \eta}=\frac{d S}{d \eta} & =0 \\
\left(\frac{d^{2}}{d \eta^{2}}-1\right) \frac{d H}{d \eta} & =0
\end{aligned}
$$




$$
\frac{d \omega}{d \eta}+\left(\frac{d^{2}}{d \eta^{2}}-1\right) H=0
$$

Equations (13e) and (17) then yield

$$
\left.\begin{array}{c}
S=1, \quad C=-\alpha^{-2} \varepsilon^{-1}, \quad \theta=\omega^{-1}, \\
\omega=\beta \alpha^{-2} \varepsilon^{-1}(2 \log \alpha \varepsilon)^{\frac{1}{2}} \eta, \quad H=\beta \alpha^{-3} \varepsilon^{-1}(2 \log \alpha \varepsilon)^{\frac{1}{2}}[1-\exp (-\eta)] .
\end{array}\right\}
$$

In the thermal layer, we define $t=z / \gamma$ as the variable in this layer, where $\gamma$ is the thickness of the layer. It is assumed that

$$
\gamma \ll \alpha^{-1}
$$

We then find from (7) and (19), after applying matching conditions (matching the solutions to the corresponding solutions in the intermediate layer), that the governing equations reduce to

$$
\frac{d^{4} \omega}{d t^{4}}=\frac{d^{2} H}{d t^{2}}=\frac{d C}{d t}=\frac{d^{2} S}{d t^{2}}=0
$$

and

$$
\frac{d^{2} \theta}{d t^{2}}+(1-\omega \theta) \omega=0
$$

where it is found that we should have the following conditions

$$
F R \gamma^{2} D^{2}=1 \text { and } D=\beta \alpha^{-1} \varepsilon^{-1} \gamma(2 \log \alpha \varepsilon)^{\frac{1}{2}} .
$$

Equation (20) then yields

$$
\omega=D t, \quad H=\alpha^{-1} D t, \quad C=-\alpha^{-2} \varepsilon^{-1},
$$

$S=1 \quad$ and $\quad \theta=\frac{t}{2 D} \int_{0}^{1}\left(1-\hat{t}^{2}\right)^{-1 / 4} \exp \left(-\frac{t^{2} \hat{t}}{2}\right) d \hat{t}$

To determine $F$, we evaluate the expression $\left\langle|\nabla \theta|^{2}\right\rangle$ and $\left\langle(1-\overline{\omega \theta})^{2}\right\rangle$ in (4) and, after a formal procedure to maximize $F$ (Chan, [2]), we find that

$$
\begin{aligned}
& F=\left(4 I^{-1}\right)^{4 / 3} 3^{-3} R^{\frac{1}{2}} Q^{-1 / 3}\left(\log T a R^{-1}\right)^{1 / 3}, \\
& \gamma=\left(I 4^{-1}\right)^{1 / 3} 3 R^{-\frac{1}{2}} Q^{1 / 3}\left(\log T a R^{-1}\right)^{-1 / 3}, \\
& \alpha=(R / 9)^{1 / 4}, \quad \text { where } I=1.062 .
\end{aligned}
$$

The conditions (14), (15) and (19) yield the following results:

$$
\begin{aligned}
T a & =O\left(Q R^{\frac{1}{2}}\right), \\
R^{\frac{1}{2}} & \ll Q \ll R^{3 / 4} \log R,
\end{aligned}
$$




$$
R \ll T a \ll R^{5 / 4} \log R .
$$

(iii) The range $R^{3 / 4}(\log R) \ll Q \ll(R \log R)^{4 / 5}$. The boundary layer structure discussed in the previous range was based essentially on the condition that

$$
\alpha \leqslant R^{1 / 4} \ll \gamma^{-1} .
$$

It was found indeed that the value of $\alpha$ which maximizes $F$ is proportional to $R^{1 / 4}$. Now, as $Q$ further increases beyond the range (24b), we have a new condition that

$$
\alpha \leqslant \gamma^{-1} \leqslant R^{1 / 4}
$$

Consequently, $F$ is now maximized by the largest possible value of $\alpha$. These results indicate that there exists a new boundary layer solution for the case in which $y=O\left(\alpha^{-1}\right)$. The interior and the inner layer of this new boundary layer structure are the same as those discussed in the previous range. In the thermal layer (which is now merged with the intermediate layer of thickness $\left.\alpha^{-1}\right)$, we define $\eta=z / \delta$ as the layer variable. We find from (7), after applying matching conditions (matching the solutions to the corresponding solutions in the inner layer), that (17) holds and that $\theta$ must be found as the solution of

$$
\left(\frac{d^{2}}{d \eta^{2}}-1\right) \theta+(1-\omega \theta) \omega=0
$$

for which $\theta=0$ at $\eta=0$ and $\theta \sim \eta^{-1}$ as $\eta \rightarrow \infty$. The solutions for $S, C, H$ and $\omega$ are given in (18), and the solution for (27) is found to be

$$
\theta=\frac{\eta}{2 D} \int_{0}^{1}(1+t)^{-\frac{1}{2}} \exp \left(-\frac{\eta^{2} t}{2}\right) d t
$$

To determine $F$, we use the same procedure as before and find that

$$
\begin{aligned}
& \alpha=(2 J)^{-1} R Q^{-1}\left[\log \left(R T a^{-3 / 4}\right)\right], \\
& F=J^{-2} R Q^{-1}\left[\log \left(R T a^{-3 / 4}\right)\right],
\end{aligned}
$$

where $J=1.77$.

The present analysis assumes that

$$
\alpha^{-1} \ll T a^{\frac{1}{2}} \alpha^{-3} \ll 1
$$

Using (14b), (25), (29) and (30), we find the following results

$$
\begin{gathered}
T a=O\left[R^{2} Q^{-1}\left(\log R T a^{-3 / 4}\right)^{2}\right], \\
R^{3 / 4}(\log R) \ll Q \ll(R \log R)^{4 / 5}, \\
(R \log R)^{6 / 5} \ll T a \ll R^{5 / 4}(\log R) .
\end{gathered}
$$


(iv) The range $(R \log R)^{4 / 5} \ll Q \ll R$. For $Q$ larger than the order of $(R \log R)^{4 / 5}$, (30) is no longer valid, and we must have a new condition in which

$$
\alpha^{-1} \ll \delta \ll T a^{\frac{1}{2}} \alpha^{-3}
$$

The boundary layer structure consists of four regions : the interior, the inner layer of thickness $T a^{\frac{1}{2}} \alpha^{-3}$, the thermal layer, and a thinner layer of thickness $\alpha^{-1}$. The interior and the inner layer are essentially the same as those discussed in the range (24b). In the thermal layer of thickness $\delta$, we define $\zeta=z / \delta$ as the layer variable. We find from (7) and (32), after matching the solutions to the corresponding solutions in the inner layer, that

$$
\frac{d C}{d \zeta}=\frac{d S}{d \zeta}=\frac{d H}{d \zeta}=0, \quad \alpha \delta^{2} H=\frac{d \omega}{d \zeta}, \quad \alpha^{2} \theta=F R(1-\omega \theta) \omega
$$

and

$$
F R D_{1}^{2}=\alpha^{2}, \text { where } D_{1}=\beta \alpha^{2} \delta\left[2 T a^{-1} \log \left(T a^{\frac{1}{2}} \alpha^{-3} \delta^{-1}\right)\right]^{\frac{1}{2}}
$$

The solutions are

$$
\left.\begin{array}{c}
H=D_{1} \delta^{-1} \alpha^{-2}, \quad \omega=D_{1} \zeta, \quad \theta=\left[D_{1}\left(1+\zeta^{2}\right)\right]^{-1} \zeta, \\
S=1 \quad \text { and } C=-\alpha^{-2} \varepsilon^{-1} .
\end{array}\right\}
$$

The solutions for $\omega, \theta, S$ and $C$ satisfy the required boundary conditions at $\zeta=0$. A thinner layer is then needed to adjust the solution to satisfy the correct boundary condition on $H$. This is a layer of thickness $\alpha^{-1}$ with $\eta=z \alpha$ as its variable. It is then a simple matter to find that

$$
H=D_{1} \delta^{-1} \alpha^{-2}[1-\exp (-\eta)] .
$$

The maximization of $F$ with respect to $\alpha$ proceeds as before, and we find that

$$
\begin{aligned}
F & =\left(\frac{2}{\pi^{2}}\right) R Q^{-1}\left(\log R T a^{-2 / 3}\right), \\
\delta & =\left(\frac{\pi}{2}\right) R^{-1} Q\left(\log R T a^{-2 / 3}\right)^{-1}, \\
\alpha & =2.29 Q^{1 / 4}, \\
T a & =O\left(Q^{3 / 2}\right), \\
(R \log R)^{4 / 5} & \ll Q \ll R .
\end{aligned}
$$




\subsection{Rotation is dominant}

The solutions for this case are essentially those already discussed in Section 3.3, except the differences to be given below in each of the three different ranges for $T a$ :

(i) The range $1 \ll T a \ll R$. The heat flux $Q$ must be in the range $1 \ll Q \ll T a^{\frac{1}{2}}$.

(ii) The range $R \ll T a \ll R^{11 / 10}\left(\log T a R^{-1}\right)$. The heat flux $Q$ must be in the range $1 \ll Q \ll \operatorname{TaR}^{-\frac{1}{2}}$.

(iii) The range $R^{5 / 4}(\log R) \ll T a \ll R^{3 / 2}$. The heat flux $Q$ must be in the range $1 \ll Q \ll T a R^{-\frac{1}{2}}$.

\section{Discussion}

The problem of combined effects of rotation and magnetic field on thermal convection presents some very unexpected features. The linear theory (Chandrasekhar [4]) predicts, for example, that the critical Rayleigh number for the onset of linear instability, for certain ranges of $T a$ and $Q$, has two minima. Also, the critical wave number for the onset of convection decreases discontinuously for $Q$ less than some critical value $Q_{c}$. Thus for $Q$ near $Q_{c}$, cells of two very different sizes will appear simultaneously. The results of the nonlinear problem studied in this paper present some more unexpected features. The results in the range (ii) of Section 3.5 indicate that the magnetic effects are marginally dominant, since, for $T a$ larger than the order of $Q R^{\frac{1}{2}}$, rotation replaces the field and becomes dominant. Therefore, for $T a$ greater than some critical value $T a_{c}$ (given $R$ and $Q$ ), the expressions for $F$ and $\alpha$ given by $(23 \mathrm{a}, \mathrm{c})$ change discontinuously to the expressions given in the range (ii) of Section 3.3, with $\alpha$ suddenly increasing and $F$ decreasing. However, it must be noted that this feature holds only for $\mathrm{Ta}$ in the range (ii) of Section 3.3. For Ta outside this range and $T a \gg Q R^{\frac{1}{2}}$ there is no solution because the single mode analysis of thermal convection with rotation alone (I) does not predict any boundary layer solution in the range $R^{11 / 10}\left(\log T a R^{-1}\right) \ll T a \ll R^{5 / 4}(\log R)$. The feature discussed above can also be stated in the following form. For $T a$ about the critical value $T a_{c}$. wave numbers and heat fluxes of two different values will be predicted simultaneously. As $T a$ increases and passes through $T a_{c}$, the horizontal scale of the cells suddenly decreases, and the magnetic field indicates that it can facilitate convection. The results in the range (iv) of Section 3.5 also indicate that the magnetic effects are marginally dominant since, for $T a$ larger than the order of $Q^{3 / 2}$, rotation replaces the field and becomes dominant. Thus, for $T a$ greater than some critical value $T a_{c}$ (given $R$ and $Q$ ), the expressions for $F$ and $\alpha$ given by (36a, c) change discontinuously to the expressions given in the range (iii) of Section 3.3, with $\alpha$ and $F$ suddenly increasing. However, it must be noted that this feature holds only for $T a$ in the range (iii) of Section 3.3. For $T a$ outside this range and $T a \gg Q^{3 / 2}$ there is no solution because 
there is no boundary layer structure for the nonlinear convection with rotation alone (I) in the range for $\mathrm{Ta}$ given above. The feature described here can also be stated in the following form. For $T a$ about the critical value $T a_{c}$, wave numbers and heat fluxes of two very different values (qualitatively) will be predicted simultaneously. As $T a$ increases and passes through $T a_{c}$, the horizontal scale of the cells suddenly decreases, and rotation indicates that it can facilitate convection.

The boundary layer structure for the case in which rotation is dominant is very much similiar to the corresponding structure for the case in which the magnetic field is dominant. In the former case, rotation first becomes important in the inner layer of thickness $E_{r}=T a^{\frac{1}{2}} \alpha^{-3}$. In the latter case, the field first becomes important in the inner layer of thickness $E_{m}=Q^{\frac{1}{2}} \alpha^{-2}$. When $E_{r} \gg E_{m}$, rotation is dominant, the inner layer is of thickness $E_{r}$ and the $E_{m}$ layer disappears. For $E_{m} \geqslant O\left(E_{r}\right)$, the field is dominant, the inner layer is now of thickness $E_{m}$ and the $E_{r}$ layer either disappears or merges with the $E_{m}$ layer. It is interesting to note that while rotation cannot become marginally dominant, the magnetic field can become marginally dominant. The reason lies in the special form of the Lorentz force in the equations of motion which is essential in determining the asymptotic solutions in the $E_{m}$ layer, even for the special case in which the Coriolis force is of comparable order of magnitude to that of the Lorentz force.

Van der Borght and Murphy [11] considered the combined effect of rotation and magnetic field on finite amplitude thermal convection using the mean field approximation. They assumed the flow is laminar and has a single mode only. They investigated three special cases: (A) $Q=O(1), T a=O(1)$, (B) $Q=O(1), T a \gg 1$, and (C) $T a=O(1), Q \gg 1$. For case A, they find that $F$ is proportional to $R^{1 / 3}$, just as in the nonrotation case. However, the proportionality constant depends on $Q, T a$ and $\alpha$ and decreases as $Q$ or Ta increases. Cases B and $C$ are just the cases in which rotation alone and the field alone are dominant, respectively. Their general result that an increase in the field strength can facilitate convection is in agreement with the present study.

\section{References}

[1] F. H. Busse, "On the mean field problem of thermal convection", Max-Planck Inst. Phys. Astrophys. Rep. MPI-PAE/Astro 31 (1970), 1-31.

[2] S. K. Chan, "Infinite Prandtl number turbulent convection", Studies in Appl. Math. 50(1971), 13-49.

[3] S. K. Chan, "Investigation of turbulent convection under a rotational constraint", J. Fluid Mech. 64 (1974), 477-506.

[4] S. Chandrasekhar, Hydrodynamic and hydromagnetic stability (Oxford Univeristy Press, 1961), Chapter 5.

[5] J. R. Herring, "Investigation of problems in thermal convection", J. Atmos. Sci. 20 (1963), 325-338.

[6] L. N. Howard, "Notes from summer study program in geophysical fluid dynamics", Woods Hole Oceanographic Inst. 1 (1965). 
[7] C. Hunter and N. Riahi, "Nonlinear convection in a rotating fluid", J. Fluid Mech. 72 (1975), 433-454.

[8] N. Riahi, "Upper-bound problem for a rotating system", J. Fluid Mech. 81 (1977), 523-528.

[9] N. Riahi, "Some predictions from the mean-field equations of magneto-convection", Aust. J. Phys. (1980) (in press).

[10] R. Van der Borght, J. O. Murphy and E. A. Spiegel, "On magnetic inhibition of thermal convection", Aust. J. Phys. 25 (1972), 703-718.

[11] R. Van der Borght and J. O. Murphy, "The combined effect of rotation and magnetic field on finiteamplitude thermal convection", Aust. J. Phys. 26 (1973), 617-643.

Department of Earth and Space Sciences

Institute of Geophysics and Planetary Physics

University of California

Los Angeles

California 90024

U.S.A. 